Actes du 16e colloque du GERAS

\title{
Le caméscope, instrument de convergence
}

\section{Pierre Vuillaume}

\section{OpenEdition \\ Journals}

Édition électronique

URL : http://journals.openedition.org/asp/3967

DOI : 10.4000/asp.3967

ISSN : 2108-6354

\section{Éditeur}

Groupe d'étude et de recherche en anglais de spécialité

\section{Édition imprimée}

Date de publication : 1 décembre 1995

Pagination : 389-405

ISSN : 1246-8185

\section{Référence électronique}

Pierre Vuillaume, "Le caméscope, instrument de convergence », ASp [En ligne], 7-10 | 1995, mis en ligne le 16 novembre 2013, consulté le 03 mai 2019. URL : http://journals.openedition.org/asp/3967 ; DOl : 10.4000/asp.3967

Ce document a été généré automatiquement le 3 mai 2019.

Tous droits réservés 


\title{
Le caméscope, instrument de convergence
}

\author{
Pierre Vuillaume
}

1 Une expérience que je suis en train de mener m'a semblé entrer dans le thème du XVI colloque du GERAS, «L'anglais de spécialité : convergences - divergences » en particulier lorsque j'ai lu, dans les premières lignes de la lettre de Michel Perrin et Robert Marret du 10 octobre 1994: « ... convergences de cette discipline (l'anglais) et de l'autre' vers l'apprenant...».

2 En effet, en concertation avec le directeur d'une toute nouvelle Maîtrise de notre Faculté, j'étudie l'utilisation du caméscope comme «catalyseur de la performance en expression orale $»^{1}$. Je me propose donc d'expliquer la démarche qui a abouti, pour le Professeur Émile-Jean Vincent et moi-même, à la définition de nos objectifs. Je parlerai ensuite de la mise en œuvre de ces objectifs, mise en œuvre que j'illustrerai par de courts extraits du travail fait par les étudiants.

3 Je ferai ensuite une analyse globale des résultats obtenus et des questions que ces résultats soulèvent. C'est auprès des étudiants, acteurs de l'expérience, que je chercherai certaines réponses à ces questions, avant d'évoquer, pour finir, les suites données à la première partie de l'expérience et les réponses, sans doute plus précises, qu'elles peuvent apporter.

\section{Définition des objectifs, les protagonistes de l'expérience}

\subsection{Le professeur de chimie}

4 Président de la Commission de Chimie de la Faculté des Sciences de Saint-Jérôme, Université Aix-Marseille 3, Responsable, entre autres, des Relations Internationales Scientifiques de cette Université, le Professeur Vincent est depuis le mois de septembre 1993 Directeur de la MST « Génie des Transformations de la Matière et Environnement ». 
C'est en cette qualité qu'il s'est adressé au Service de Laboratoires de Langues de la Faculté pour faire assurer un enseignement d'anglais à ses étudiants.

L'objectif primordial qu'il souhaitait que nous nous fixions était l'entraînement à l'expression orale, associé, dans la mesure du possible, à un entrânement à la compréhension orale.

6 En effet, nommé Responsable des Relations Internationales Scientifiques de l'Université il y a quatre ans, et requis à tout moment de donner son avis, il a été, selon ses propres termes, "traumatisé par son incapacité à traduire ses connaissances et ses revendications " au cours de "conversations liées directement à sa profession », par exemple lors de discussions sur des équivalences de programmes, ou à propos de conditions d'attribution de bourses Erasmus. Il n'avait jusqu'alors été confronté qu'à l'anglais de sa spécialité, soit écrit, soit utilisé lors de conférences et congrès internationaux, sans rencontrer de réelles difficultés.

7 Au cours de ces quatre années, cependant, il s'est « rendu compte qu'il pouvait parler, même mal », et il insiste sur ce dernier point. Si bien que l'objectif numéro un qu'il visait pour nos étudiants était de "leur enlever une espèce d'inhibition à s'exprimer en anglais", et plus précisément de "les mettre dans cette situation tendue qu'est la participation à une conversation scientifique et de politique scientifique ».

\subsection{Le professeur d'anglais}

8 De mon côté, titulaire depuis le mois de novembre 1993 du DEA de Langue anglaise des spécialités scientifiques et techniques de l'Université de Bordeaux 2 après avoir effectué un travail de recherche sur l'utilisation d'une émission radio de la BBC, Science in Action, comme instrument de progrès en compréhension orale pour un groupe d'élèvesingénieurs faux-débutants, j'avais décidé de poursuivre ma recherche dans le domaine, cette fois, de l'expression orale, tout en gardant les documents authentiques de la BBC comme base de travail, après que l'idée eut été lancée, au cours d'une des soutenances de la session 1993 du DEA, d'étudier dans quelle mesure le caméscope pouvait être un " catalyseur de la performance en expression orale ».

9 Le calendrier a voulu que le début des cours de la MST soit prévu pour le mois de janvier 1994. Je pouvais ainsi entreprendre sans tarder mon expérience, dont j'exposai les grandes lignes au Professeur Vincent. Cette recherche répondait à sa propre attente.

10 Nous avons naturellement eu un certain nombre d'entretiens, formels ou informels. Par ailleurs, le Professeur Vincent a répondu trois fois à mon invitation et est venu sur place voir ce que faisaient nos étudiants. Il a chaque fois exprimé le point de vue du scientifique sur le contenu de la prestation à laquelle il venait d'assister, tout en insistant auprès d'eux sur l'importance qu'il attache à la nécessité de rendre leur propos accessible au grand public.

\subsection{La $1^{\text {re }}$ promotion de la Maîtrise GTME : troisième protagoniste}

11 Il s'agit de 24 étudiants, répartis en 2 groupes de 12, qui reçoivent en première année un enseignement de 30 heures d'anglais, à raison de 2 heures par semaine. L'idée originale, propre à cette Maîtrise, est de rassembler deux options, Chimie et Géologie, pour aborder 
les problèmes liés à l'environnement. En seconde année il est aussi prévu d'assurer 30 heures de cours.

12 À la suite de tests de placement, j'ai constitué deux groupes rigoureusement identiques dans leur composition. Le cadre et l'esprit de la collaboration entre ces trois protagonistes étant connus, je vais maintenant présenter l'expérience elle-même.

\section{Mise en œuvre}

\subsection{Les moyens}

Dans notre service nous disposons de laboratoires de langues, de magnétophones, bien sûr, d'un caméscope, et de magnétoscopes. Grâce à la réception satellite, nous pouvons recevoir, entre autres, les programmes radio de la BBC World Service.

\subsection{Organisation des cours}

14 Le programme prévu est le même pour les deux groupes. Les documents utilisés sont extraits de deux émissions radio de la BBC: Science in Action, émission de vulgarisation abordant divers domaines scientifiques, et Global Concerns, assurée par la même équipe que la précédente, mais traitant uniquement de sujets liés à l'environnement.

Pour un document donné, nous suivons les différentes étapes destinées à développer la compréhension orale du document, puis une tâche précise est donnée à préparer. Par exemple, après avoir étudié un entretien sur les incendies et leurs conséquences sur l'érosion en Californie, les étudiants ont dû préparer un débat sur le même sujet, mais cette fois en Provence.

16 Les étudiants sont divisés, dans chaque groupe, en trois équipes de quatre. Le débat ou l'entretien doit durer entre trois et cinq minutes. Dans le groupe dit «Audio », le débat est enregistré au magnétophone; dans le groupe dit "Vidéo», il est enregistré au caméscope.

17 Les critères d'évaluation (voir Annexe 1) sont préalablement distribués et commentés. Ils sont les mêmes dans chaque groupe pour tout ce qui touche au contenu de l'entretien, à sa structure, et à ses aspects linguistiques et phonologiques. Une partie de la note totale $(1 / 10)$ est affectée aux caractéristiques plus spécifiques du magnétophone ou du caméscope.

18 Ainsi, toutes choses étant égales, à l'exception des moyens d'enregistrement des prestations, les résultats doivent faire apparaître la part que l'un ou l'autre instrument peut éventuellement jouer dans la qualité des performances orales des étudiants.

19 Notre propos et notre recherche visant plus précisément le caméscope, je me propose de commenter quelques courts extraits des prestations filmées faites au cours de cette première période.

\section{Illustration}

Les contraintes de temps m'obligeant, cela va de soi, à faire un choix dans les deux heures et demie d'enregistrements que nous avons réalisés dans le groupe Vidéo (une durée 
équivalente a été réalisée dans le groupe Audio), j’ai décidé de suivre une de nos étudiantes au fil des étapes de notre travail. Pourquoi cette jeune fille en particulier? Parce que dès le premier jour, avant même que chacun ait pris sa place, apprenant qu'elle venait de «tomber » dans le groupe Vidéo, qui, de plus, commençait à huit heures, elle s'est déclarée « doublement pénalisée »... Il aurait été tentant de choisir quelqu'un ayant un préjugé favorable, ou de sélectionner des extraits des meilleures prestations : il m'a semblé que l'influence du caméscope serait plus nettement perceptible à travers les réactions d'une personne hostile au départ.

21 Je signale au passage qu'en fin de parcours j'ai demandé à tous les étudiants de préciser s'ils m'autorisaient ou non à montrer des extraits de leurs prestations, dans le cadre strict de l'analyse et du compte-rendu de notre travail. Tous ont donné leur accord signé.

\subsection{The Map}

22 Premier contact avec la caméra. Pour qu'il se fasse sans que les étudiants se sentent directement impliqués, je suis les conseils de Yves Bourron et Jean Deneville (1991) en demandant de commenter brièvement une carte que les étudiants découvrent. Il s'agit d'une carte du National Geographic portant uniquement sur l'environnement.

Le résultat est un certain embarras, qui se traduit par l'attitude physique, repliée sur soi, et la brièveté des interventions de Barbara.

Sandrine: We can see a map of the world, with the... different parameters of pollution... We can read it.

Stéphane: Population pressure, air pollution... ozone concern... concerns, acid rain, and so on...

Sandrine: Mm... The back... Barbara, can you read, er..., different point ?

Barbara: (Laughter) No. I can't read.

Stéphane: She hasn't got his glasses. (Laughter from Barbara)

Sandrine: Natural rates of change... in Peru and Minnesota for the forest; and

different parameters of population, population projection and population

explosion...

Teacher: Barbara?

Barbara: I can't read...

Teacher: Well, thank you.

\subsection{Gunpowder: BBC \& New Scientist}

Il s'agissait cette fois de comparer la façon dont un même sujet avait été traité à la radio et dans un magazine scientifique. Les étudiants disposaient d'une grille précisant les différents points de comparaison dont la fonction était de faire apparaître les spécificités de chaque document, mais aucune directive n'avait été donnée quant à la façon de présenter les choses. On va voir, dans l'extrait qui suit, que si l'anglais est correct dans l'ensemble, les attitudes sont figées, les regards évitent la caméra, les protagonistes n'ont pas de vrais échanges, et l'impression produite est celle d'une litanie.

Barbara: In New Scientist, err... Guy Fawkes used gunpowder to try to blow up Parliament in sixteen five.

Eric: So, now, what do you reckon to the commercial condi... consideration?

Stéphane: So, on BBC interview, err... We can see all sorts of uses of gunpowder, err..., we can see that gunpowder is used to make signalling rockets, maroons, flares, quarrying of slates, and err.., they are mainly examples of uses ,err..., in a commercial way. 
Barbara: In the New Scientist, we can see that the ingredients of the new product are available in Britain, while gunpowder has be... has to be imported from Germany.

\subsection{New Chemistry for a Better Environment}

L'extrait qui suit va mettre en évidence un certain nombre de points : les quatre étudiants ont préparé un scénario, et ils jouent des rôles, même s'ils ont choisi de garder leurs noms. En effet, c'est à la suite d'une suggestion directe d'Yves Bourron que j'ai bâti les débats que les étudiants devaient préparer autour de jeux de rôles, ce qui a eu pour effet de les libérer. On le voit notamment chez Barbara, qui s'exprime avec une certaine aisance, emploie assez naturellement un certain nombre d'expressions propres à la communication, tout en n'évitant pas certains pièges liés en particulier au vocabulaire.

Sandrine: Well, I see a question in the audience.

Barbara: Good morning. Barbara Olive, from France. I'd like to ask a question to Doctor Alazard, because I'm not really sure if I'd go along with him on what he said. Doctor, you talk about a new process, totally safe food... safeful for nature, but are you really sure that your products won't create prob... environmental problems in a few years like CFC actually?

\subsection{AAAS in San Francisco: a spontaneous interview}

À la suite d'un exercice de compréhension orale sur un récent congrès de l'American Association for the Advancement of Science à San Francisco les étudiants étaient censés avoir assisté au congrès, y avoir fait une communication, et répondre aux questions d'un journaliste de la $\mathrm{BBC}$ sur cette communication dont ils étaient les seuls à décider du contenu. Cette fois l'entretien était individuel, et l'exercice était destiné à voir comment les étudiants réagissaient à ce que le Professeur Vincent appelle volontiers une " agression ", c'est-à-dire comment ils réussissaient à exprimer un point de vue et à se faire comprendre.

Nous allons voir Barbara lutter contre sa réserve naturelle, tout en faisant face à la situation et en jouant le jeu. Le sujet qu'elle avait choisi n'était pas un sujet facile, et elle a réussi à en parler. Il faut noter que, contrairement aux entretiens préparés en équipe, cette prestation fait apparaître chez cette étudiante, comme chez l'ensemble de la promotion, une détérioration des éléments grammaticaux et phonologiques de son discours. C'est, semble-t-il, le prix à payer pour la spontanéité, et la façon de résoudre ce problème précis reste une préoccupation.

Barbara: Well, er..., I'm a -let me introduce me. I'm, er..., a doctor of, er..., the (slight laughter) (PV : "Yes? ")... I'm a doctor of the organization Doctors of the World, and I want to speak to you of the problem of the AIDS in, er..., the Third World.

Reporter: H'm, h'm...

Barbara: It's the same thing that, the..., the démographie problem ; er..., we t..., we talked about in the meeting of the American Ass..., Association for the Adv..., Advancement of Science in San Francisco.

Reporter: $\mathrm{H}^{\prime} \mathrm{m}, \mathrm{h}$ 'm...

Barbara: I want to..., talk to the..., TV for..., for saying, er..., at the..., at the politics to give more money to -for the research. If we don't find a vaccine for, er..., against the..., the AIDS, in a few years it will be a disaster in Africa. 


\subsection{Fires and Erosion in California}

L'intérêt de l'extrait suivant est de mettre en évidence la capacité d'invention dont ont fait preuve les étudiants au fur et à mesure que notre travail avançait, associée à un sens de l'humour et à un goût du jeu favorisés, de toute évidence, par le fait que la prestation était filmée. Dans le cas présent, les problèmes liés aux incendies et à l'érosion ont bien été traités, il faut le préciser. Barbara, devenue présentatrice, n'a plus vraiment peur de la caméra.

Barbara: Good afternoon. Welcome to our program Friends of the Forest. Today, our subject is the problem of fire and erosion in California. I'm very glad to receive Mrs Sandrine Marchand, Doctor of the University of Saint-Jérôme, Mr Eric Cantona, whose house was burnt last summer in Provence, and Mr Cousteau.

Two "supporters" of Eric Cantona rush on the set in order to get autographs... They happen to be David and Yannick..., yelping "Canto!!! Canto!!!" Some slight disorder follows..., with Stéphane saying "Good boy!", and Fabrice calling "Security!"

\subsection{No Gas on Route 66 : a critical analysis}

Je voudrais pour finir déborder légèrement sur le travail fait en seconde année pour mettre en évidence les progrès des étudiants, et... du professeur... Comme pour l'exercice sur la BBC et New Scientist, il s'agit de faire l'analyse critique d'un document (un reportage filmé sur la voiture électrique extrait de Tomorrow's World) en suivant une série de critères donnés par le professeur. Pas de litanie cette fois, mais un véritable débat, présenté avec clarté, dans une langue soignée, bien que non exempte d'erreurs.

Barbara: Good morning! Following the diffusion of the report "No Gas on Route $66 "$ ", we carried out an opinion poll in order to know how successful this coverage was. There are three main opinions. So, we have invited three persons to illustrate this opinion poll.

Miss Marchand and Mr Boyer didn't really appreciate this report. On the contrary, Stéphane Alazard, you seem to have enjoyed, er, this program.

Stéphane: Yes. The topic was very interesting. I could discover the running of electric cars, which was totally unknown to me.

Barbara: Sorry to interrupt you, but Miss Marchand doesn't seem to be... agree with you...

Sandrine: From my point of view, they didn't talk sufficiently about the technical aspects of these cars.

Et le groupe Audio? Un échantillon équivalent à celui qui précède montrerait la même évolution. Faute de temps, c'est à travers les résultats obtenus par les étudiants dans les deux groupes que nous allons pouvoir en juger.

\section{Résultats}

31 Si dans un premier temps nous comparons les résultats obtenus par chaque groupe pour une prestation donnée, celle sur Industrial Revolutions par exemple, on voit clairement que les performances des étudiants, dans les domaines qu'ils ont en commun (structure et contenu d'une part, langue d'autre part), évalués suivant les mêmes critères, ces performances sont équivalentes (voir graphiques Annexe 2). 
- Aucune en ce qui concerne l'anglais, profitable sur le facteur moi.

- Important, car cela a demandé beaucoup de travail et aussi beaucoup d'attention.

- Être obligé de jouer un rôle, faire attention au langage (anglais).

- Mise en évidence des fautes.

- Le caméscope m'a fait prendre conscience de tout ce qui est tenue physique (dû au stress) et du niveau de la difficulté pour communiquer en anglais.

- Voir ses défauts, ses erreurs...

- Permet de connaître nos capacités, d'avoir une opinion de soi par nous-mêmes, mais aussi par les autres.

- Progression dans la compréhension orale. Plus à l'aise pour s'exprimer, en particulier devant une caméra.

- Importance de se voir, visualiser son comportement.

- A permis de me voir parler anglais, donc de voir mes défauts.

- Comme précédemment, le caméscope permet de rendre un meilleur résultat du travail (et aussi de s'amuser!)

\section{Groupe Audio}

- Efforts, volonté de donner vie aux textes.

- Le magnétophone permet d'améliorer la prononciation et l'aisance à parler.

- On a tendance à s'appliquer.

- Sur les résultats d'anglais :“???" Mais face à un micro : très positif !

- S'habituer à entendre sa voix.

- Ça nous permet de nous rendre compte de nos défauts. (... et qualités !)

- Apprendre à s'écouter pour mieux analyser ses erreurs. 
- L'écoute de son travail a posteriori permet une approche plus critique et corrective.

- Libération lors de l'expression orale.

- Une amélioration récente en lecture, donc en compréhension

- S'entendre parler révèle ce que les autres entendent quand on s'adresse à eux et nous pousse à faire des efforts pour mieux nous faire comprendre.

- Cela améliore notre "accent" et nous oblige à tenir un discours cohérent en anglais.

Question b. Le regard que vous jetez sur l'année écoulée fait-il apparaître un bilan POSITIF ? NÉGATIF ? Comment l'expliquez-vous?

\section{Groupe Vidéo}

- La mise en œuvre de la méthode vidéo y est pour beaucoup. C'était très stimulant d'utiliser cette technique, car j'ai le sentiment qu'elle nous rapproche beaucoup des problèmes auxquels nous serons confrontés en pratique.

- Progrès et amélioration de l'audition.

- Évolution, et travail d'équipe intéressant.

- Amélioration de la compréhension et phonétique.

- Bonne ambiance. Aucune peur de nos erreurs.

- C'était très agréable.

- La mise en œuvre des prestations est inhabituelle et est assez stimulante.

Groupe Audio

41 - Toujours le travail en groupe et l'obligation de parler.

- Ça change des cours souvent trop scolaires, donc ennuyants.

- Tout le monde a pu participer. Personne n'est resté muet dans son coin.

- Être obligé de prendre la parole implique de faire attention à ses erreurs.

- Ambiance conviviale ; travail de groupe ; sujets intéressants.

- Positif mais doit mieux faire (pour moi) ; une nouvelle façon de travailler.

- On a insisté sur l'oral, ce qui est primordial.

Le troisième extrait de notre questionnaire va toutefois indiquer une tendance en faveur du caméscope dans la mesure où, comme nous allons le voir, les étudiants du groupe Vidéo ne souhaitent pas que leurs activités soient modifiées, alors que certains du groupe Audio manifestent un certain désir de découverte.

Question c. Souhaitez-vous que les groupes actuels (composition et activités spécifiques) soient reconduits en seconde année ? Oui ou Non

Groupe Vidéo : OUI pour activités, sans importance pour composition. (NDLR : = « Garder le travail au caméscope, même si les équipes doivent changer. »)

Si vous avez répondu NON, quelles modifications souhaitez-vous apporter en ce qui concerne : Leur composition? Leurs activités spécifiques? (Pas de commentaires)

Groupe Audio

46 - OUI : Pas de caméra!

- NON :

- C'est intéressant de changer d'activités et de composition même si j'ai apprécié les gens avec qui j'ai travaillé.

- Composition sans importance ; personnellement, j'aimerais faire de la vidéo.

- On voudrait bien le caméscope.

- Composition, «never mind! » Activités : pourquoi ne pas inverser les activités? 
- Composition : rien ; activités : faire les deux : magnétophone et caméscope.

- Faire moitié/moitié pour comparer.

\section{Prolongements}

La toute dernière remarque rejoignait en fait mes projets pour la seconde année, à savoir :

- demander à l'ensemble des étudiants de faire une prestation enregistrée uniquement au magnétoscope ;

- demander à l'ensemble des étudiants de faire une prestation enregistrée au caméscope, à partir d'un document tiré de la télévision anglaise ;

- pour introduire une part de création spontanée dans le cadre de débats, désormais devenus familiers, faire tirer au sort, à l'intérieur de chaque équipe, des rôles sur un sujet préalablement étudié en cours ;

- parallèlement, enfin, demander à chaque équipe de préparer un reportage, audio ou vidéo, sur un sujet de son choix.

- Privilégier le caméscope, car c'est un moyen plus complet.

- Vidéo, car on a plus de liberté (variantes).

- Vidéo : en quelque sorte, c'est une préparation à un entretien d'école ou en entreprise.

- Vidéo : on peut s'aider par des gestes.

- Il vaut mieux privilégier la vidéo, qui mêle image et son.

- Privilégier la vidéo, car elle allie l'image et la parole.

- Vidéo : cela motive plus pour faire un scénario plus original ; on essaye de ne pas lire.

- Aucun moyen à privilégier. Travailler les deux.

- Les deux ; on a deux approches différentes avec ces modes de travail.

- Les deux, ça dépend des avis de chacun: l'utilité est de pouvoir revenir sur nos prestations et de voir ce qui ne va pas!

\section{Groupe Audio}


- Je préfère la vidéo, car on peut plus « jouer » avec son personnage.

- Personnellement, je préfère la vidéo.

- Le caméscope, car cela nous force à maîtriser nos paroles et nos expressions.

- Il faudrait privilégier le caméscope. C'est un outil d'avenir et il permet de gommer certains « tics »?

- Aucun, les deux sont complémentaires.

- Tout dépend de ce que l'on veut : pour le contenu : la radio; pour la mise en scène : la télé.

- Pas de moyen privilégié : chacun a son avantage et son inconvénient.

- Les deux expériences sont à vivre de façon égale, car elles permettent la mise en valeur du langage (audio) et une plus grande souplesse de l'expression (vidéo).

\section{BIBLIOGRAPHIE}

Achard J.-P. 1991. Des images et des sons : théorie et technique. Paris : Éditions Eyrolles.

Bourron Y. et J. Denneville. 1991. Se voir en vidéo : pédagogie de l'autoscopie. Paris : Les Éditions d'Organisation.

Brown J. D. 1988. Understanding Research in Second Language Learning: A teacher's guide to statistics and research design. Cambridge : Cambridge University Press.

Cooper R., M. Lavery et M. Rinvolucri. 1984. Video. Oxford : Oxford University Press.

Hadli C. 1992. L'évaluation, règles du jeu : des intentions aux outils. Paris : ESF Éditeur.

Lonergan J. 1984. Video in Language Teaching. Cambridge : Cambridge University Press.

Narcy J.-P. 1990. Apprendre une langue étrangère. Didactique des langues : le cas de l'anglais. Paris : Les Éditions d'Organisation.

\section{ANNEXES}

\section{Annexe 1}


NOM:

PRENOM:

DATE:

M. S. T. GENIE DES TRANSFORMATIONS DE LA MATIERE ET ENVIRONNEMENT
CRITERES D'EVALUATION : EXPRESSION ANALOGIQUE : (DEBATS OU ENTRETIENS)

\begin{tabular}{|l|l|l|l|l|}
\hline & OUI NON Nh de Pls \\
\hline 1. L'ATTITUDE GENERALE (8 points) &
\end{tabular}

1.1. Le corps est decontracté, souple, lintervenant est détendu.
$(=$ pas de manifestation de malaise, d'anxiété ou de nervosité)

1.2. Lintervenant est toume vers lauditoire ou vers linterlocuteur.

1.3. Les bras sont ouverts, en direction de linterlocuteur ou de l'auditoire.

1.4. Le visage est détendu, expressif, souriant.
$(=$ pas de mouvements nerveux incontróles, ou de visage sombre, ou las $)$

2. LA VoIX (3 points)

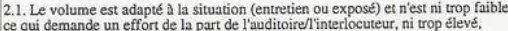

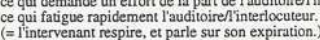

2.2. Le débit (rapport des mots śnoncés dans un termps donne) est r $r$ gulté
$(=$ permet de recevoir clairement le message ; nest pas trop important).

2.3. Laarticulation est claire.

3. LES PAUSES ET LES SILENCES ( 3 points)

3.1. Significatifs

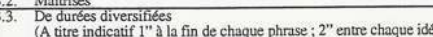

(A titre indicatif 1" 1 la fin de ching
$3^{\prime \prime}$ avant un propos important)

4. LE REGARD (3 points)

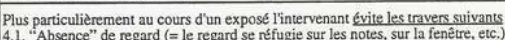

4.2. "Faux" regard (= lintervenant fait semblant de regarder, il efleure lefles audite

5. L'ECOUTE (2 points)

On est attentif a la question posée. On est attentif a la réponse qui est donnéce

On suit ce qui se dit, même sil lon n'intervient pas directemen
On se tourne vers la camera si 'lon doit s'adresser au public.

6. LES NOTES PERSONNELLES ( 2 points)

Lintervenant connât parfaitement son sujet tet se lihère ainsi de ses noues

TOTAL / 20

Faculte des Sciencess - Marseille St. Jetône
LABORATOIRE DE LANGUES (2)

\section{Annexe 2}

\begin{tabular}{|c|c|c|}
\hline NOM: & PRENOM: & DATE \\
\hline
\end{tabular}

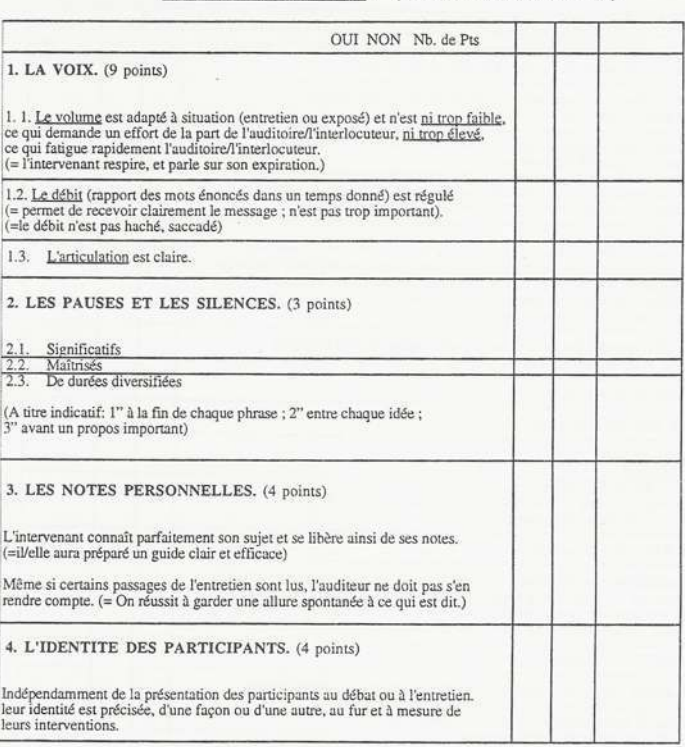

TOTAL / 20:

Faculte des Sciencess - Marseille St. Jetorme
LABORATOIRE DE LANGUES @

Annexe 3 


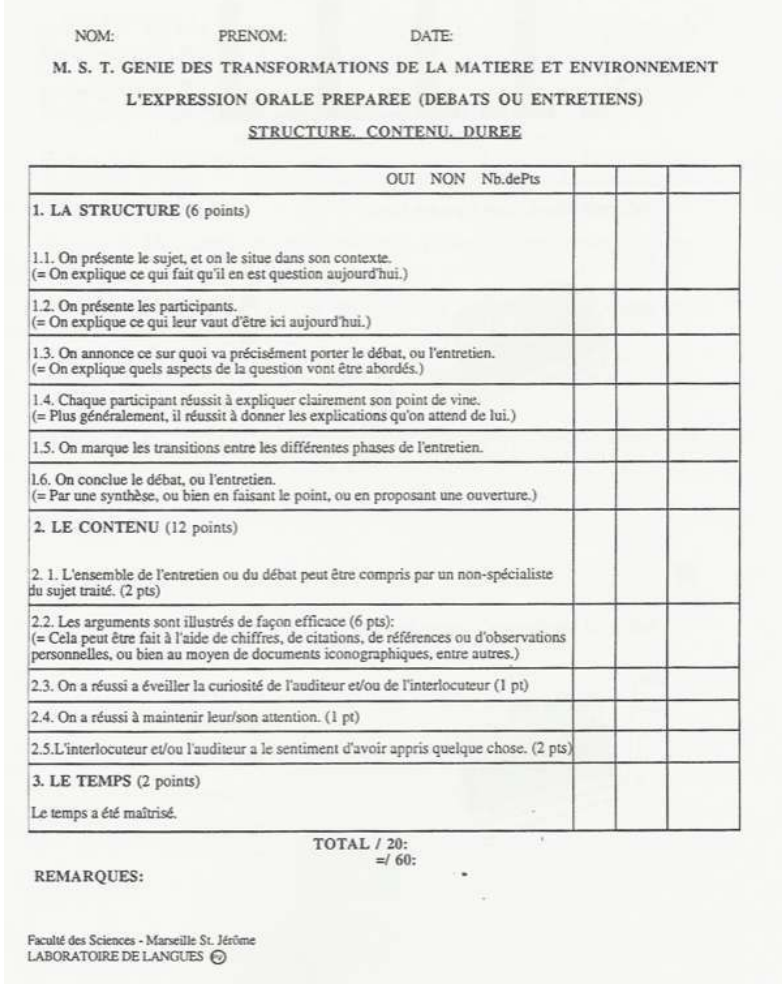

\section{Annexe 4}
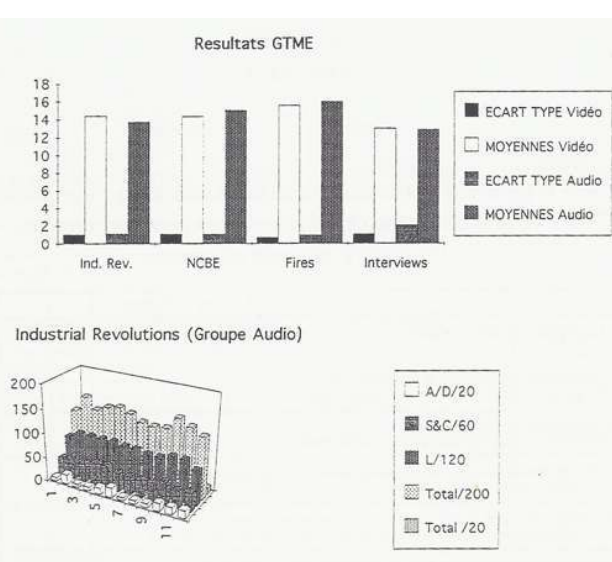

Industrial Revolutions (Groupe Vidéo)

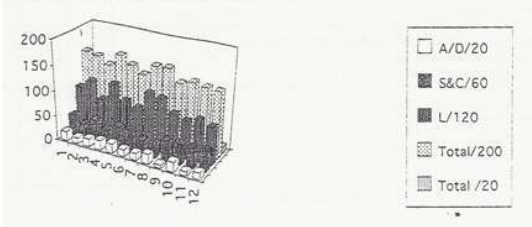

\section{NOTES}

1. Ce travail fait l'objet d'une thèse soutenue à Bordeaux en décembre 1995. 


\section{RÉSUMÉS}

Une étude menée, avec des étudiants de maîtrise, sur l'utilisation du caméscope comme catalyseur de la performance en expression orale a donné lieu à une série d'échanges entre le professeur d'anglais qui a mené cette étude et le directeur de la Maîtrise, par ailleurs Président de la Commission de Chimie de l'Université. L'objet de cette communication est de montrer comment la recherche effectuée par le linguiste semble répondre aux objectifs que s'est fixés le scientifique, et de retracer les étapes de cette convergence : démarche ayant abouti, pour chacun des protagonistes, à la définition des objectifs et à la mise en œuvre.

A study on the use of the camcorder as a catalyst of oral performance was carried out with a group of undergraduate students. Their head, who is also the head of the Chemistry Department of the University, and their English teacher exchanged views on the subject on a number of occasions. This article explains how the linguist's research answered the scientist's expectations. It focuses on the stages of that convergence: each protagonist's definition of his objectives and how they were implemented.

INDEX

Mots-clés : caméscope, expression orale, évaluation, vidéo

Keywords : camcorder, oral expression

\section{AUTEUR}

\section{PIERRE VUILLAUME}

Pierre Vuillaume enseigne à la Faculté des sciences et techniques de Saint-Jérôme, Université Aix-Marseille 3. pierre.vuillaume@sfr.fr 